Mirzayeva G., Rzayeva V.

\title{
ANALYSIS OF FREE OSCILLATIONS OF A NON-HOMOGENEOUS PIPE ALONG THICKNESS AND LENGTH, TAKING INTO ACCOUNT THE RESISTANCE OF THE EXTERNAL ENVIRONMENT
}

Structural elements made of heterogeneous natural and artificial materials are widely used in the construction of modern building complexes and in many other areas. Among them is the use of boards and shells of different configurations. Currently, one of the most important requirements for designers and accountants is to properly assess the mechanical properties of the material of the structural element and the impact of the environment in contact during operation. Taking these into account, the mathematical solution of the problem becomes difficult, and if not, serious mistakes can be made. One of the most problematic places is considering the resistance of the external environment.

The objects of research are modern pipes exposed to the external environment.

Pasternak model, which is one of the mathematical models that accurately reflects the elastic real properties of the environment, Winkler model, which is characterized by two constants, the model of Karnet model and the model of Rjanitsin model are analysed.

In the course of the research, the method of separation of variables and then Bubnov-Galerkin method is used, which explain relationship between the dimensionless value of frequency, the parameters that characterize the non-homogeneous of the base, and the pipe. The selection of special frequencies is carried out by selecting the corresponding special equation and boundary conditions. There are nonlinear algebraic equations and their solution using computer technology. It is shown that when the mechanical properties of the pipe vary in length, the above solution method does not work and the determination of the characteristic parameters must be performed using other approximate analytical methods. In engineering practice, it is usually sufficient to find the basic tone of the frequency. As a result of the research it is shown that the external environment effects are important for non-homogeneous pipe and should be considered in the design of the structure-ground interaction. In the future, the proposed approach and should be considered in the design of the structure non-homogenous pipe interaction.

Keywords: non-homogeneous pipe, environment with complex properties, Winkler model, Pasternak model, Karnet model.

Received date: 04.05.2020

Accepted date: 10.06 .2020

Published date: 31.10 .2020
Copyright (C) 2020, Mirzayeva G., Rzayeva V. This is an open access article under the CC BY license (http://creativecommons.org/licenses/by/4.0)

\section{Introduction}

It is known that modern pipelines for various purposes are operated in natural conditions with complex properties and are complex engineering structures. During the design and construction of pipelines, engineers are required to properly assess the properties of the pipeline (technological process, welding stresses, non-uniform composition, and properties of the substrate that are strongly dependent on natural conditions).

It is noted that taking into account these, it is difficult to determine the frequency-amplitude characteristics, and if errors are made.

Given that explosions, earthquakes, strong winds, and other causes can cause pipelines to vibrate [1,2].

Recently, in engineering practice, pipes made of nonhomogeneous materials made of natural and non-homogenous materials are widely used and are operated in various natural conditions and exposed to external influences. As a result of inhomogeneity, its elastic modulus and density in materials are constantly changing. The most common non-homogeneous bases are those characterized by two constants and having viscous elastic properties.

Nowadays modern pipelines for various purposes are operated in natural conditions with complex properties and are complex engineering structures research are relevant. Thus, objects of research are modern pipes exposed to the external environment. The aim of this article is learn the relationship between the dimensional value of the frequency, the parameters that characterize the non-homogenous of the base, and the pipe.

\section{Methods of research}

It is assumed that a straight section of the pipeline is located in an environment with complex properties. 
In engineering practice, the following mathematical models of the reaction of the external environment to natural conditions and other reasons are used [3-5].

1. Winkler model:

$$
q=k w,
$$

where $q$-reaction of the environment; $k\left(N / m^{3}\right)$ - Winkler coefficient; $w-$ a bend.

2. Pasternak model:

$$
q=k_{1} w-k_{2} \frac{d^{2} w}{d x^{2}},
$$

where $k_{1}$ - Winkler and $k_{2}\left(\mathrm{~N} / \mathrm{m}^{3}\right)$ - Pasternak coefficient.

3. Rjanitsin model:

$$
q=k_{1} w+k_{2} \frac{d^{2} w}{d t^{2}},
$$

where $k_{2}$ characterizes the viscosity; $t$ - time.

4. Karnet model (non-homogeneous):

$$
q=k_{1}(x) w+k_{2}(x) \frac{d^{2} w}{d t^{2}},
$$

where $k_{1}(x)$ and $k_{2}(x)$ are involuntary environmental characteristics and are determined experimentally.

\section{Research results and discussion}

It is considered that, modulus of elasticity $E$ of the pipe material and density $\rho$ - thickness and length vary continuously [6]:

$$
E=E_{0} f_{1}(x) f_{2}(z) ; \rho=\rho_{0} \psi_{1}(x) \psi_{2}(z),
$$

where $E_{0}, \rho_{0}$ - corresponds to a homogeneous material; $f_{1}(x)$ the function itself and the derivative up to the second-order is an uninterrupted function.

Since the thickness of the pipe is a heterogeneous, non-homogeneous pipe, the neutral axis does not coincide with the midline, i. e.:

$$
\int_{-h}^{h} \sigma_{1} \mathrm{~d} z=0, M=\int_{-h}^{h} \sigma_{1} z \mathrm{~d} z
$$

(6) in the system $e-z_{0} \chi=0$.

The condition and the expression for the moment can be written as follows:

$$
M=E_{0} J_{0} A f(x) \frac{\partial^{2} w}{\partial x^{2}} .
$$

Considering (7), the equations of motion for each foundation can be written as follows:

$$
\begin{aligned}
& E_{0} J_{0} A \frac{\partial^{2}}{\partial x^{2}}\left[f_{1}(x) \frac{\partial^{2} w}{\partial x^{2}}\right]+\bar{\rho} \psi_{1}(x) \frac{\partial^{2} w}{\partial t^{2}}+k_{1} w=0 \\
& E_{0} J_{0} A \frac{\partial^{2}}{\partial x^{2}}\left[f_{1}(x) \frac{\partial^{2} w}{\partial x^{2}}\right]+k_{1} w-k_{2} \frac{\partial^{2} w}{\partial x^{2}}+\bar{\rho} \psi_{1}(x) \frac{\partial^{2} w}{\partial t^{2}}=0 ; \\
& E_{0} J_{0} A \frac{\partial^{2}}{\partial x^{2}}\left[f_{1}(x) \frac{\partial^{2} w}{\partial x^{2}}\right]+k_{1} w+\left(k_{2}+\bar{\rho} \psi_{1}(x)\right) \frac{\partial^{2} w}{\partial t^{2}}=0 ; \\
& E_{0} J_{0} A \frac{\partial^{2}}{\partial x^{2}}\left[f_{1}(x) \frac{\partial^{2} w}{\partial x^{2}}\right]+k_{1}(x) w+\left(k_{2}(x)+\psi_{1}(x)\right) \frac{\partial^{2} w}{\partial t^{2}}=0, \\
& \bar{\rho}=\rho_{0} h \int_{-l / 2}^{l / 2} \Psi_{2}(z) \mathrm{d} z .
\end{aligned}
$$

As can be seen from the above equations, the latter case covers the most general case.

The fourth case needs to be investigated in detail. The goal here, at the same time to take into account that the base is a non-homogeneous viscous elastic, taking into account the non-uniformity of the pipe.

As can be seen, the equation of motion is a differential equation with complex special derivative and its $f(x), \psi_{1}(x)$ It is difficult to determine the exact solution at arbitrary prices. Let's solve the equation using approximate analytical methods. In the first stage let's use the method of separation into variables, in the next stage Bubnov-Galerkin method [7, 8].

In the first stage, let's look for the function as follows:

$$
w(x, t)=w_{0}(x) e^{i \omega t},
$$

where $\omega_{0}$ - must meet border conditions; $\omega$ - frequency. Substituting the expression (9) in the fourth equation of (8); $w_{0}(x)-$ obtain the usual derivative linear equation with respect to:

$$
\begin{aligned}
& f_{1}(x) \frac{d^{4} w_{0}}{d x^{4}}+2 f^{I}(x) \frac{d^{3} w_{0}}{d x^{3}}+f^{I I}(x) \frac{d^{2} w_{0}}{d x^{2}}+ \\
& +\overline{k_{1}}(x) w_{0}-\omega^{2}\left(\overline{k_{2}}(x)+\overline{\rho_{0}} \psi_{2}(x)\right) \omega_{0}=0 .
\end{aligned}
$$

The following symbols have been adopted here:

$$
\begin{aligned}
& \overline{k_{1}}(x)=k_{1}(x)\left(E_{0} J_{0} A\right)^{-1} ; \\
& \overline{k_{2}}(x)=k_{2}(x)\left(E_{0} J_{0} A\right)^{-1} ; \\
& \rho_{1}=\overline{\rho_{0}}\left(E_{0} J_{0} A\right)^{-1} .
\end{aligned}
$$

Since equation (10) is complex, let's solve it by the Bubnov-Galerkin orthogonalization method and look for the solution as follows:

$$
w_{0}(x)=\sum_{i=1}^{n} a_{i} \theta_{i}(x)
$$

where $a_{i}$ - unknown constants; $\theta_{i}-$ each of them must meet the border conditions.

Error function (10) and (11) taking into account is written as follows:

$$
\eta(x)=\sum_{i=1}^{n} a_{i}\left[\begin{array}{l}
f_{1}(x) \frac{d^{4} \theta_{i}}{d x^{4}}+2 f^{I}(x) \frac{d^{3} \theta_{i}}{d x^{3}}+ \\
+f^{I I}(x) \frac{d^{2} \theta_{i}}{d x^{2}}+\overline{k_{1}}(x) \theta_{i}- \\
-\omega^{2}\left(\overline{k_{2}}(x)+\overline{\rho_{0}} \psi_{2}(x)\right) \theta_{i}
\end{array}\right] \neq 0 .
$$

The orthogonalization condition is written as follows [9]:

$$
\int_{0}^{l} \eta(x) \theta_{q}(x) \mathrm{d} x=0, q=\overline{1, n} .
$$

In arbitrary approximation $\omega^{2}(13)$ the algebraic line is determined from the system equation. Since the system consists of a system of homogeneous equations, the main determinant must be zero in the absence of its trivial solution $[6,10]$ :

$$
\left\|\omega^{2}\right\|=0 \text {. }
$$

Equation (14) is a nonlinear algebraic equation and its solution does not cause any difficulties using computer 
technology. In engineering practice, they are usually content with finding the basic tone of the frequency, i. e.:

$$
\int_{0}^{l} \eta(x) \theta_{1}(x) \mathrm{d} x=0
$$

Conditionally paid and $\omega^{2}$ is appointed:

$$
\omega^{2}=\frac{\int_{0}^{l}\left[f_{1}(x) \frac{d^{4} \theta_{1}(x)}{d x^{4}}+2 f^{I}(x) \frac{d^{3} \theta_{1}(x)}{d x^{3}}+f^{I I}(x) \frac{d^{2} \theta_{1}(x)}{d x^{2}}+\overline{k_{1}}(x) \theta_{1}(x)\right] \theta_{1}(x) \mathrm{d} x}{\int_{0}^{l}\left(\overline{k_{2}}(x)+\overline{\rho_{0}} \Psi_{2}(x)\right) \theta_{1}^{2}(x) \mathrm{d} x} .
$$

From to (16) equation when $k_{2}=0$, the solution of a similar problem is obtained for a pipe located on a nonhomogeneous foundation:

$$
\omega_{1}^{2}=\frac{\int_{0}^{l}\left[f_{1}(x) \frac{d^{4} \theta_{1}(x)}{d x^{4}}+2 f^{I}(x) \frac{d^{3} \theta_{1}(x)}{d x^{3}}+f^{I I}(x) \frac{d^{2} \theta_{1}(x)}{d x^{2}}+\bar{k}_{1}(x) \theta_{1}(x)\right] \theta_{1}(x) \mathrm{d} x}{\rho_{1} \int_{0}^{l} \psi_{2}(x) \theta_{1}^{2}(x) \mathrm{d} x},
$$

from formulas (16) and (17) can be obtained the following relationship:

$$
\left(\frac{\omega}{\omega_{1}}\right)^{2}=\bar{\omega}_{1}^{2}=\frac{\int_{0}^{l} \psi(x) \theta_{1}^{2}(x) \mathrm{d} x}{\int_{0}^{l}\left[\rho_{1}^{-1}\left(\overline{k_{2}}(x)+\psi(x)\right)\right] \theta_{1}^{2}(x) \mathrm{d} x} .
$$

If the external environment is not taken into account [4, 7]:

$$
\bar{\omega}_{21}^{2}=\frac{\int_{0}^{l}\left[f(x) \frac{d^{4} \theta_{1}}{d x^{4}}+2 f^{I} \frac{d^{3} \theta_{1}}{d x^{3}}+f^{I I} \frac{d^{2} \theta_{1}}{d x^{2}}\right] \theta_{1}(x) \mathrm{d} x}{\rho \int_{0}^{l} \psi(x) \theta_{1}^{2}(x) \mathrm{d} x} .
$$

When $\rho_{1}=\bar{\rho}_{0}$ from the above solutions only the length of $\bar{\omega}_{1}^{2}$, when $\psi(x)=1$ if only the thickness is heterogeneous, similar problems are solved.

The report was carried out for the following situations:

$$
\begin{aligned}
& k_{1}=k_{1}^{2}(1+\alpha \rho) ; k_{2}=k_{2}^{2}(1+\alpha \rho) ; \theta_{1}=\sin \pi \rho ; \\
& \psi_{1}=1+\mu \rho ; \psi_{2}=1+\varepsilon \bar{z} ; \\
& \varepsilon \in[0,1] ; \mu \in[0,1] ; \alpha \in[0,1] . \\
& \int_{0}^{1} \sin ^{2} \pi \rho d \rho=\frac{1}{2} ; \int_{0}^{1} \rho \sin ^{2} \pi \rho d \rho=\frac{1}{4} ; \int_{0}^{1} \sin 2 \pi \rho d \rho=0 .
\end{aligned}
$$

Considering the above expressions, let's obtain the following formula:

$$
\bar{\omega}_{1}^{2}=\frac{(1+0.5 \mu)}{c(1+0.5 \alpha)+(1+0.5 \mu)} .
$$

The results of the report are shown in Tables 1, 2 and Fig. 1, 2.

Tables 1, 2 calculate the value of the frequency according to the values of the coefficients characterizing the non-homogeneous plate and Fig. 1, 2 show the relationship graphs between these values and the frequencies. It is also clear from the graphs that the effect of heterogeneity was manifested by a hyperbolic change in frequency. This effect has been shown to be significant and to be taken into account in engineering design.

Table 1

Variation of frequency parameters of the non-homogeneous foundations versus: $c=0 ; \varepsilon=0$

\begin{tabular}{|c|c|c|}
\hline$\mu$ & $\bar{\omega}_{11}^{2}$ & $\bar{\omega}_{12}^{2}$ \\
\hline 0 & 1 & 1.125 \\
\hline 0.25 & 0.889 & 1 \\
\hline 0.5 & 0.8 & 0.900 \\
\hline 0.75 & 0.727 & 0.818 \\
\hline 1 & 0.666 & 0.75 \\
\hline
\end{tabular}

Table 2

Variation of frequency parameters of the non-homogeneous foundations versus: $c=1 ; \alpha=0 ; \varepsilon=0$

\begin{tabular}{|c|c|c|}
\hline$\mu$ & $\bar{\omega}_{31}^{2}$ & $\bar{\omega}_{32}^{2}$ \\
\hline 0 & 0.5 & 1.15 \\
\hline 0.25 & 0.471 & 1.022 \\
\hline 0.5 & 0.444 & 0.92 \\
\hline 0.75 & 0.421 & 0.836 \\
\hline 1 & 0.4 & 0.762 \\
\hline
\end{tabular}

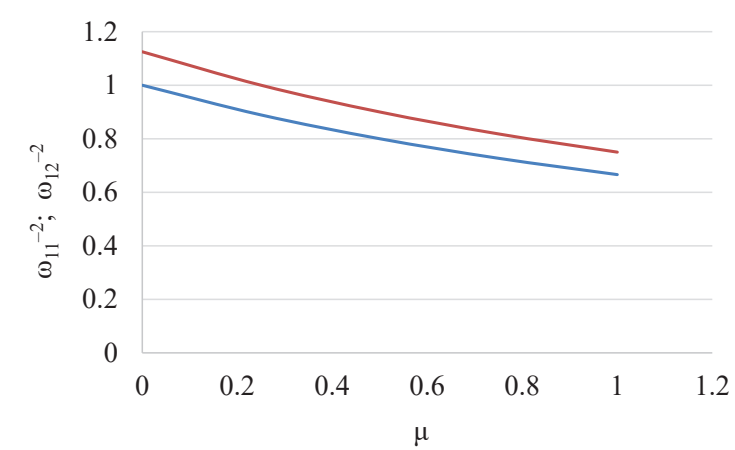

Fig. 1. The relationship between the dimensionless value of frequency, the parameters that characterize the non-homogenous of the base, and the pipe

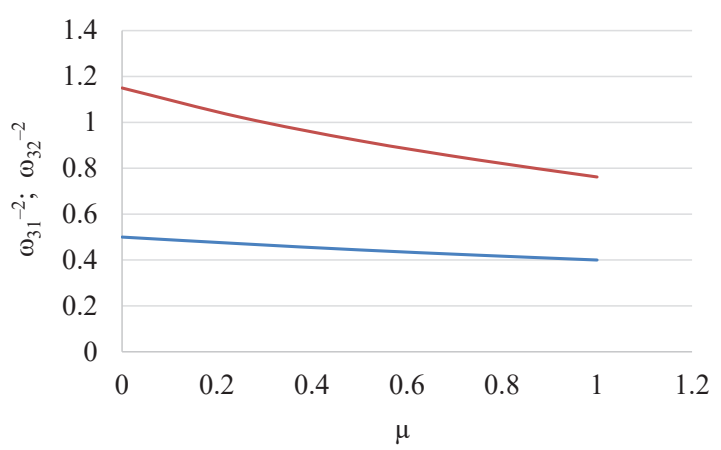

Fig. 2. The relationship between the dimensional value of the frequency, the parameters that characterize the non-homogenous of the base, and the pipe

\section{Conclusions}

Free frequency of a non-homogeneous pipe along thickness and length, taking into account the resistance of the 
external environment are analyzed. The problem of stability of the pipeline is solved and the value of the critical parameters is determined because the elastic modulus is inhomogeneous with the surface law. The solution of the problem is based on Bubnov-Galerkin method of orthogonalization. A report is made and the value of the critical force, which depends on the heterogeneity and the resistance of the base, is determined. Numerical reports are made on the specific values of the characteristic parameters and the results are presented in tables and contact curves. In the course of the research, it is obtained that the parameters that characterize the non-homogenous of the base, and the pipe so effected for materials frequency.

Nowadays, one of the most important requirements required of current designers and accountants is to properly assess the mechanical properties of the material of the structural element and the impact of the contact environment during operation. Therefore the research results will be useful for engineering designers.

\section{References}

1. Kolchin, A. S., Favarion, E. A. (1977). Teoriia uprugosti neodnorodnykh tel. Kishinev, 146.

2. Kerr, A. D. (1964). Elastic and Viscoelastic Foundation Models. Journal of Applied Mechanics, 31 (3), 491-498. doi: http:// doi.org/10.1115/1.3629667

3. Pasternak, P. L. (1954). O novom metode rascheta uprugogo osnovaniia s pomoschiu doukh fundamentnykh postoiannykh. Moscow: Gosudarstvennoe izdatelstvo literatury po stroitelstvu i arkhitekture, 1, 1-56

4. Garnet, H., Levy, A. (1969). Free Vibrations of Reinforced Elastic Shells. Journal of Applied Mechanics, 36 (4), 835-844. doi: http://doi.org/10.1115/1.3564779
5. Timoshenko, S. P. (1967). Kolebaniia v inzhenernom dele. Moscow: Nauka, 444

6. Haciyev, V. C., Mirzeyeva, G. R., Shiriyev, A. I. (2018). Effect of Winkler foundation, inhomogeneity and orthotropy on the frequency of plates. Journal of Structural Engineering $\mathcal{E}$ Ap plied Mechanics, 1 (1), 1-5. doi: http://doi.org/10.31462/ jseam.2018.01001005

7. Haciyev, V. C., Sofiyev, A. H., Mirzeyev, R. D. (1996). Free vibration of non-homogeneous elastic rectangular plates. Proceedings of the Institute of Mathematics and Mechanics, 4, 103-108.

8. Antoranz, A., Gonzalo, A., Flores, O., García-Villalba, M. (2015) Numerical simulation of heat transfer in a pipe with nonhomogeneous thermal boundary conditions. International Journal of Heat and Fluid Flow, 55, 45-51. doi: http://doi.org/10.1016/ j.ijheatfluidflow.2015.05.007

9. Haciyev, V. C., Mirzoeva, G. R., Agayarov, M. G. (2019). Free vibrations of anisotropic rectangular plate laying on a heterogeneous viscouselastic basis. Structural Mechanics of Engineering Constructions and Buildings, 15 (6), 470-476. doi: http:// doi.org/10.22363/1815-5235-2019-15-6-470-476

10. Fares, M. E., Zenkour, A. M. (1999). Buckling and free vibration of non-homogeneous composite cross-ply laminated plates with various plate theories. Composite Structures, 44 (4), 279-287. doi: http://doi.org/10.1016/s0263-8223(98)00135-4

Mirzayeva Gulnar, PhD, Senior Researcher, Department of Theory of Elasticity and Plasticity, Institute of Mathematics and Mechanics National Academy of Sciences of Azerbaijan, Baku, Republic of Azerbaijan, e-mail: gulnarmirzayeva@gmail.com, ORCID: http:// orcid.org/0000-0003-0362-3492

Rzayeva Vusala, Department of Wave Dynamics, Institute of Mathematics and Mechanics National Academy of Sciences of Azerbaijan, Baku, Republic of Azerbaijan, e-mail: rzayevavusale01@gmail.com, ORCID: http://orcid.org/0000-0001-8669-1612 